\title{
Photonic crystal fibers as a platform for pure-quartic solitons
}

\author{
Stefani, Alessio; Lo, Chih Wei; De Sterke, C. Martijn; Blanco-Redondo, Andrea
}

Published in:

Nonlinear Optics 2017

Link to article, DOI:

10.1364/NLO.2017.NTu3B.2

Publication date:

2017

Document Version

Peer reviewed version

Link back to DTU Orbit

Citation (APA):

Stefani, A., Lo, C. W., De Sterke, C. M., \& Blanco-Redondo, A. (2017). Photonic crystal fibers as a platform for pure-quartic solitons. In Nonlinear Optics 2017 [NTu3B.2] Optical Society of America (OSA).

https://doi.org/10.1364/NLO.2017.NTu3B.2

\section{General rights}

Copyright and moral rights for the publications made accessible in the public portal are retained by the authors and/or other copyright owners and it is a condition of accessing publications that users recognise and abide by the legal requirements associated with these rights.

- Users may download and print one copy of any publication from the public portal for the purpose of private study or research.

- You may not further distribute the material or use it for any profit-making activity or commercial gain

- You may freely distribute the URL identifying the publication in the public portal

If you believe that this document breaches copyright please contact us providing details, and we will remove access to the work immediately and investigate your claim. 


\title{
Photonic Crystal Fibers as a Platform for Pure-Quartic Solitons
}

\author{
Alessio Stefani ${ }^{1,2}$, Chih-Wei Lo ${ }^{3}$, C. Martijn de Sterke ${ }^{3}$, Andrea Blanco-Redondo ${ }^{3}$ \\ 1. Institute of Photonics and Optical Sciences (IPOS), School of Physics, The University of Sydney, Sydney, NSW, 2006, Australia \\ 2. DTU Fotonik, Department of Photonics Engineering, Technical University of Denmark, DK-2800 Kgs. Lyngby, Denmark \\ 3. Centre for Ultrahigh bandwidth Devices for Optical Systems (CUDOS), Institute of Photonics and Optical Science (IPOS), School of Physics, \\ The University of Sydney, Sydney, NSW 2006, Australia \\ alessiostefani@sydney.edu.au
}

\begin{abstract}
We design a photonic crystal fiber suitable for generating the intriguing pure-quartic solitons, so far only demonstrated in silicon photonic crystal waveguides. This platform will enable deeper understanding and applications of this class of solitons.

OCIS codes: (190.5530) Pulse propagation and temporal solitons; (060.5295) Photonic crystal fibers; (190.4370) Nonlinear optics, fibers
\end{abstract}

Solitons are a unique class of pulses, which propagate unchanged because of the balance between dispersion and nonlinearities. Generally, the self-phase modulation (SPM) is compensated by the negative group velocity dispersion (GVD). The perturbation of this conventional behavior by higher orders of dispersion has been extensively studied $[1,2]$. Particularly, the study of conventional solitons perturbed by fourth-order dispersion (FOD) gave rise to the field of quartic solitons $[3,4]$. Instead of being just a perturbation of standard solitons, FOD can be the leading dispersion mechanism compensating SPM, giving rise to a different class of soliton: the pure-quartic soliton (PQS) [5]. This class of solitons has a peculiar Gaussian-like shape close to its center, and an advantageous energy scaling that allows for shorter pulse durations and higher power in laser systems [4]. The first observation of PQSs was in a silicon photonic crystal waveguide with an ad-hoc engineered dispersion [5]. In this system, two-photon absorption limits the power in the waveguide and free-carriers strongly disturb the PQS dynamics. In order to fully take advantage and study the properties of PQSs, a different, more suitable platform is needed. The natural solution seems to be optical fibers since the nonlinearity is close to the ideal Kerr law and the experimental techniques are well-established. Of all optical fibers, photonic crystal fibers (PCFs) allow for the most degrees of freedom in dispersion engineering [6,7], giving the best chances to obtain the unique dispersion features required for the generation of PQSs. An optical fiber supporting PQSs would demonstrate that PQSs are not limited to one specific platform (silicon photonic crystals), but that they are a general nonlinear optical phenomenon. Moreover, the low loss of optical fibers and the existing fiber devices allow to investigate the basic physics and behavior of PQSs. Aside from such fundamental studies, optical fibers will also be an ideal platform for applications such as high power soliton lasers. We report here the results of the numerical investigation demonstrating that PCF structures can be designed to support pure-quartic solitons.
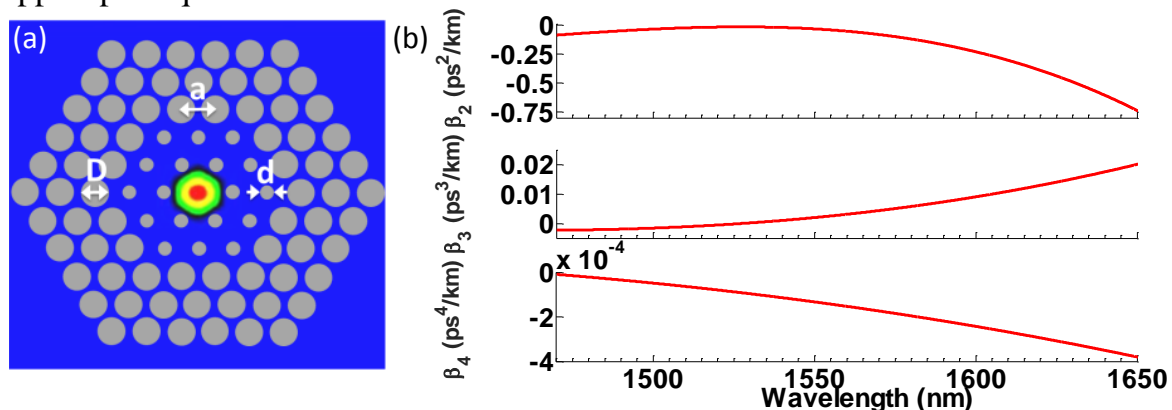

Fig. 1. (a) Investigated fiber structure and calculated fundamental mode @ 1550nm. The structural parameters optimized for are: pitch a, small holes diameter $\mathrm{d}$ and large holes diameter $\mathrm{D}$. (b) Calculated second, third and fourth order dispersion respectively from top to bottom.

We simulated the PCF structures by using the CUDOS Microstructured Optical Fiber (MOF) solver [8], which uses the multipole method to calculate the effective index [9,10]. In order to observe PQSs we require a fiber with dominant FOD, which needs to be negative, and which has near-zero GVD and third order dispersion (TOD). This requirement has to hold for a sufficient bandwidth to accommodate ultra-short pulses. Given these constraints, we investigated fiber designs similar to ultra-flattened dispersion PCF [7]. Such fibers have small holes close to the core and larger holes in the outer rings. To simplify the fabrication process we chose to use only two sizes of holes and a constant center to center distance of the holes (pitch) across the entire structure. Various combinations of number of rings with small holes and large holes were simulated. Based on these results we focused on a structure with 2 rings 
of small holes and 3 rings of large holes, as shown in Fig. 1(a). This structure allows for sufficient flexibility in dispersion tailoring and low confinement loss. Figure 1a shows the selected fiber design and the calculated mode at $1550 \mathrm{~nm}$. The operating wavelength range is around $1535-1565 \mathrm{~nm}$. The fiber has an effective area of about $14 \mu \mathrm{m}^{2}$ in this wavelength range, and the total loss is on the same order of magnitude as material loss. The calculated second $\left(\beta_{2}\right)$, third $\left(\beta_{3}\right)$ and fourth $\left(\beta_{4}\right)$ order dispersions are shown in Fig. 1(b). While we require $\beta_{4}<0$, the sign for $\beta_{2}$ and $\beta_{3}$ is irrelevant as only FOD is needed to compensate the nonlinearities. To have an intuition of the impact of the various dispersion components, the characteristic lengths associated with each of them - defined as $\mathrm{L}_{\mathrm{i}}(\lambda)=\left(\mathrm{T}_{0}\right)^{\mathrm{i}} /\left|\beta_{\mathrm{i}}(\lambda)\right|$ - is plotted in Fig. 2(a) as function of pulse duration for $\lambda=1535 \mathrm{~nm}$. The crossing point between $\mathrm{L}_{2}$ and $\mathrm{L}_{4}$ is the pulse duration for which GVD and FOD contribute equally. Above that pulse duration GVD dominates, while below FOD dominates. This is equivalent to the blue coloring of Fig. 2(b). Figure 2(a) gives some more information about the system: for example, for a 50 fs pulse the FOD length is $L_{4}=63.3 \mathrm{~m}$, while the GVD length is $L_{2}=136.2 \mathrm{~m}$, which is more than double. Though it is currently unknown how much larger the FOD needs to be to obtain PQS, we note from [5] that PQS were generated with $\mathrm{L}_{4}=3.66 \mathrm{~L}_{2}$. From a practical point of view, Fig. 2(a) determines the length of the fibers to be fabricated, as propagation over several FOD lengths is required for a convincing observation of PQSs. On the other hand, short fibers generally suffer smaller structural fluctuations in the drawing process and therefore the dispersion tends to be closer to the one calculated by looking at a single cross-section. With these two factors in mind, we conclude that lengths on the few meters scale will be sufficient for observing PQS pulses below 20 fs duration. To conclude the feasibility study, one last criterion needs to be met for PQS generation: it is in fact necessary that the nonlinear length and the dispersion length are matched. As an example, the power required to match a 50 fs pulse is $1.9 \mathrm{~W}$ (shown by the dashed line in Fig. 2(a)). The blue area in Fig. 2(b) shows the wavelength region where the FOD dominates over both GVD and TOD as a function of the pulse duration, $\mathrm{T}_{0}$ (note that for a Gaussian pulse $\mathrm{T}_{0}=\mathrm{T}_{\mathrm{FWHM}} / 1.665$ ). The presence of such area is the necessary condition for the structure under investigation to support PQSs. Within the area we need to make sure that the FOD is dominant for the bandwidth of a pulse with a given width. The arrows in Fig. 2(b) are an indication of the bandwidth of a transformlimited Gaussian pulse centered at $1535 \mathrm{~nm}$ for various pulse durations. Clearly the FOD area is sufficiently broad to contain pulses spanning at least from 10 to $60 \mathrm{fs}$ for this particular design.

In conclusion, we demonstrated a PCF structure having the necessary dispersion, across a sufficient bandwidth for the generation and investigation of pure-quartic solitons and their dynamics. The proposed structure, which fulfills the criteria for fabrication in terms of cross-sectional dimensions and length (investigation of tolerances was also carried out, but is not reported here), will be soon fabricated allowing the investigation of PQS in this platform.
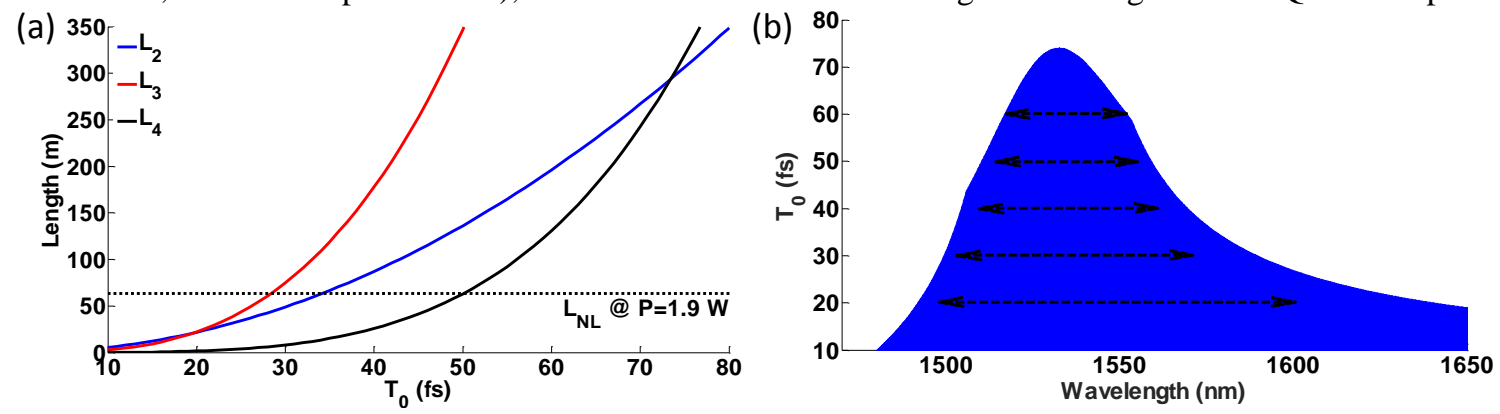

Fig. 2. (a) Characteristic lengths associated with second, third and fourth order dispersion @ 1535 nm as a function of the pulse duration. (b) Wavelength region where FOD is dominating as a function of the pulse duration $\mathrm{T}_{0}$. The black arrows indicate the bandwidth of transform limited Gaussian pulses for the various time durations.

\section{References}

[1] A. Höök, M. Karlsson, "Ultrashort solitons at the minimum-dispersion wavelength: effects of fourth-order dispersion," Opt. Lett. 18, 13881390 (1993).

[2] N. Akhmediev, A.V. Buryak, M. Karlsson, "Radiationless optical solitons with oscillating tails," Opt. Commun. 110, 540-544 (1994).

[3] S. Roy, F. Biancalana, "Formation of quartic solitons and a localized continuum in silicon-based waveguides," Phys. Rev. A 87, 025801 (2013).

[4] I. Christov, M.N. Murnane, H.C. Kapteyn, J. Zhou, C.P. Huang, “Fourth-order dispersion-limited solitary pulses,” Opt. Lett.19, 1465-1467 (1994).

[5] A. Blanco-Redondo, C.M. de Sterke, J.E. Sipe, T.F. Krauss, B. Eggleton, C. Husko, "Pure-quartic solitons,” Nature Comm. 7, 10427 (2016).

[6] P.St.J. Russell, "Photonic-Crystal Fibers," J. Lightwave Technol. 24, 4729-4749 (2006)

[7] K. Saitoh, M. Koshiba, T. Hasegawa, E. Sasaoka, "Chromatic dispersion control in photonic crystal fibers: application to ultra-flattened dispersion," Opt. Express 11, 843-852 (2003)

[8] sydney.edu.au/science/physics/cudos/research/mofsoftware.shtml

[9] T.P. White, B.T. Kuhlmey, R.C. McPhedran, D. Maystre, G. Renversez, C.M. de Sterke, L.C. Botten, "Multipole method for microstructured optical fibers. I. Formulation,” J. Opt. Soc. Am. B 19, 2322-2330 (2002)

[10] B.T. Kuhlmey, T.P. White, C.M. de Sterke, D. Maystre, G. Renversez, R.C. McPhedran, L.C. Botten, "Multipole method for microstructured optical fibers II: Implementation and results," J. Opt. Soc. Am. B 19, 2331-2340 (2002). 